\title{
Ảnh hưởng của cảm nhận về trách nhiệm xã hội và lãnh đạo đạo đức đến ý định nghỉ việc của nhân viên văn phòng tại Thành phố Hồ Chí Minh
}

\section{The effects of perceptions of CSR and Ethical Leadership on office worker's intention turnover in Ho Chi Minh City}

\author{
Nguyễn Thị Bích Châm ${ }^{*}$, Vũ Trung Kiên ${ }^{1}$ \\ ${ }^{1}$ Trường Đại học Kinh tế Thành phố Hồ Chí Minh, Việt Nam \\ *Tác giả liên hệ, Email: chamng@gmail.com
}

THÔNG TIN

DOI: $10.46223 / \mathrm{HCMCOUJS}$.

econ.vi.15.3.1332.2020

Ngày nhận: 05/12/2019

Ngày nhận lại: 31/12/2019

Duyệt đăng: 21/01/2020

\section{Tì khóa:}

cảm nhận về trách nhiệm xã hội, lãnh đạo đạo đức, sự tham gia công việc, sự kiệt sức, ý định nghỉ việc

Keywords:

corporate social responsibility perceptions, ethical leaderships, work engagement, burnout, turnover intentions

\section{TÓM TẮT}

Nghiên cứu ảnh hưởng của cảm nhận về Trách nhiệm xã hội và Lãnh đạo đạo đức đến Ý định nghỉ việc của nhân viên văn phòng tại Tp. Hồ Chí Minh, nhằm giúp cho doanh nghiệp hiểu rõ hơn tác động của các nguồn lực tâm lý đến cảm nhận của người lao động là nhân viên văn phòng, để từ đó đưa ra các gợi ý nhằm giảm ý định nghỉ việc của nhân viên văn phòng. 248 nhân viên văn phòng đang làm việc tại thành phố Hồ Chí Minh đã tham khảo sát. Dữ liệu thu thập được từ cuộc khảo sát được gạn lọc và đưa vào phân tích bằng phần mềm SPSS 25 và $\mathrm{AMOS} 22$. Kết quả nghiên cứu đã chứng minh Trách nhiệm xã hội và Lãnh đạo đạo đức ảnh hưởng tích cực đến Sự tham gia công việc. Lãnh đạo đạo đức ảnh hưởng tiêu cực đến Sự kiệt sức còn Trách nhiệm xã hội thì không. Ý định nghỉ việc của nhân viên bị tác động tiêu cực bởi Sự tham gia công việc và tích cực bởi Sự kiệt sức.

ABSTRACT
This research studies how White-collar Employees'
Corporate Social Responsibility perception and Ethical
Leadership influence Turnover intention in Ho Chi Minh City.
The purpose of the study is to help the organization understand
the impact of philosophy resource on employees' perception and
give managerial implication to reduce turnover intention as well
as improve employee brand value. Data were collected through
the Survey on 248 white-collar employees in Ho Chi Minh City
and be filtered and input in the software named SPSS 25 và
AMOS 22. The empirical findings show CSR and Ethical
leadership are both related to work engagement positively.
Ethical leadership negatively affects work engagement but CSR
does not. Turnover intention is affected by work engagement
negatively and burnout positively.




\section{Giới thiệu}

Hiện nay, Khái niệm Ý định nghỉ việc đã được các tác giả nghiên cứu sâu, rộng với nhiều quan điểm, lĩnh vực, vùng lãnh thổ khác nhau như Chaudhary (2017), Lin và Liu (2017), Ng, Yam, và Aguinis (2018), Sarfraz, Qun, Abdullah, và Alvi (2018),... Đa số các nghiên cứu về ý định nghỉ việc tập trung vào mối quan hệ với các yếu tố cơ bản về lương thưởng, môi trường làm việc, tính chất công việc, cam kết của tổ chức,... có thể kể đến các nghiên cứu của Martin và Roodt (2008), Khan và cộng sự (2014), $\mathrm{Lu}, \mathrm{Lu}, \mathrm{Gursoy}$, và Neale (2016). Các yếu tố này tác động trực tiếp hay gián tiếp đến sự hài lòng của nhân viên và từ đó tác động đến lòng trung thành hay ý định nghỉ việc của nhân viên. Bên cạnh đó, cũng có khá nhiều nghiên cứu về ý định nghỉ việc dưới quan điểm đạo đức như: lãnh đạo đạo đức, trách nhiệm xã hội... có thể liệt kê một số nghiên cứu như Demirtas và Akdogan (2014), HollingWorth và Valentine (2014), Palanski, Avey, và Jiraporn (2014), Sarfraz và cộng sự (2018). Hầu hết các nghiên cứu kể trên đề cập từng mối quan hệ riêng lẻ giữa yếu tố lãnh đạo đạo đức hoặc trách nhiệm xã hội với ý định nghỉ việc. Vì vậy, nghiên cứu này nhằm góp phần làm rõ hơn ảnh hưởng đồng thời của trách nhiệm xã hội và lãnh đạo đạo đức với ý định nghỉ việc trong bối cảnh nghiên cứu tại Việt Nam.

Bên cạnh đó, trong các nghiên cứu gần đây tại Việt Nam các tác giả thường nghiên cứu trực tiếp khái niệm trách nhiệm xã hội và lãnh đạo đạo đức, còn trong nghiên cứu này tác giả tiếp cận hai khái niệm này qua cảm nhận của nhân viên văn phòng, nhằm giúp doanh nghiệp hiểu sâu hơn nguyên nhân dẫn đến ý định nghỉ việc của nhân viên phòng, từ đó giúp doanh nghiệp tập trung vào các nguồn lực tâm lý để có thể đưa ra các giải pháp hiệu quả cho tình trạng nhân viên văn phòng nghỉ việc nhiều như hiện nay.

\section{Lý thuyết và mô hình nghiên cứu}

\subsection{Các khái niệm nghiên cúu}

\subsubsection{Trách nhiệm xã hội và cảm nhận về Trách nhiệm xã hội}

Khái niệm Trách nhiệm xã hội đã được rất nhiều tác giả nghiên, cùng với đó đã có rất nhiều định nghĩa, quan điểm khác nhau về khái niệm này, có thể kể đến một số quan điểm về Trách nhiệm xã hội như: tác giả Carroll (1991) đưa ra khái niệm Trách nhiệm xã hội gồm 4 thành tố trách nhiệm: kinh tế, pháp lý, đạo đức và thiện nguyện; Bên cạnh đó, cũng có nhiều nghiên cứu xem xét trách nhiệm xã hội từ góc độ của các bên liên quan như Aguilera, Rupp, Williams và Ganapathi (2007), Turker (2009), Vitaliano (2010). Theo các tác giả, trách nhiệm xã hội là những hoạt động mang tính tích cực của doanh nghiệp bao gồm: tuân thủ pháp luật, các nghĩa vụ thuế, bảo vệ môi trường, ... cũng như đối với các bên liên quan như: cổ đông, đối tác, chính phủ, nhân viên, các tổ chức xã hội...

Ngoài ra, cũng có nhiều tác giả tiếp cận trách nhiệm xã hội theo cấu trúc gồm cấp độ vĩ mô (nghĩa là tổ chức) và cấp độ vi mô (tức là cá nhân). Theo đó trách nhiệm xã hội có thể được coi là hoạt động tự nguyện của công ty vì phúc lợi xã hội tạo ra kết quả ở tầm vĩ mô, thì nó cũng có thể được coi là vấn đề cấp vi mô liên quan đến kết quả của nhân viên. Vì vậy, các tác giả này đã đưa ra khái niệm cảm nhận của nhân viên (emloyee's perception) về Trách nhiệm xã hội như nghiên cứu của Ng và cộng sự (2018), Rupp và cộng sự (2018), Sarfraz và cộng sự (2018).

Với nghiên cứu của mình, tác giả sẽ sử dụng khái niệm cảm nhận về trách nhiệm xã hội của nhân viên (perceived CSR) vì đây luôn là yếu tố dự đoán về phản ứng của nhân viên (cảm xúc, thái độ và hành vi) vượt trội hơn so với trách nhiệm xã hội khách quan (objective CSR).

\subsubsection{Cảm nhận về Lãnh đạo đạo đức}

Tác giả Brown, Treviño, và Harrison (2005) là một trong những nhà nghiên cứu đầu tiên 
xem Lãnh đạo đạo đức như một lý thuyết độc lập. Theo tác giả, Lãnh đạo đạo đức được định nghĩa là sự thể hiện chuẩn mực hành vi thông thường thông qua các hành động cá nhân, các mối quan hệ giữa các cá nhân với nhau, và lãnh đạo đạo đức sẽ thúc đẩy hành vi của cấp dưới thông qua giao tiếp hai chiều là củng cố và ra quyết định. Các nghiên cứu Brown và cộng sự (2005), Brown và Treviño (2006) đã sử dụng lý thuyết học tập xã hội (Social Learning Theory) và lý thuyết trao đổi xã hội (Exchange and power in sociai life) để giải thích sự ảnh hưởng của Lãnh đạo đạo đức đến hành vi của cấp dưới. Theo quan điểm học tập xã hội, các nhà lãnh đạo đạo đức với vai trò là người làm gương đã tạo ra ảnh hưởng đến hành vi của cấp dưới. Thêm vào đó, những cấp dưới học theo những hành vi được chấp nhận bằng cách quan sát các phần thưởng và hình phạt mà các nhà lãnh đạo đạo đức thể hiện. Còn theo quan điểm trao đổi xã hội, trước tiên các nhà lãnh đạo đạo đức thể hiện sự công bằng và quan tâm đến nhân viên cấp dưới. Đến lượt mình, nhân viên cấp dưới cảm thấy bắt buộc phải đáp lại và hành động theo những kỳ vọng của nhà lãnh đạo đạo đức (Brown et al., 2005; Brown \& Treviño, 2006).

Trong nghiên cứu này tác giả xem xét ảnh hưởng của nhà lãnh đạo đức đến phản ứng của nhân viên hay nói cách khác là cảm nhận của nhân viên về phong cách lãnh đạo đạo đức của nhà lãnh đạo.

\subsubsection{Sụ tham gia công việc}

Theo các lý thuyết nghiên cứu trước đây có hai cách tiếp cận với khái niệm Sự tham gia công việc. Cách tiếp cận đầu tiên được ủng hộ bởi Maslach và Leiter (2008), các tác giả đặt Sự tham gia công việc là mặt đối lập trực tiếp với Sự kiệt sức. Đặc trưng của Sự tham gia công việc là năng lượng cao, sự tham gia cao và hiệu quả cao. Cách tiếp cận thứ hai được xuất phát từ tác giả Schaufeli, Salanov, Gonzalez-Roma, và Bakker (2002). Theo các tác giả, Sự tham gia công việc và Sự kiệt sức là hai trạng thái tâm trí tương quan tiêu cực trái ngược với nhau và kết quả là họ xác định "Sự tham gia công việc đề cập đến một trạng thái tâm trí tích cực, thỏa mãn liên quan đến công việc được đặc trưng bởi sự nhiệt huyết, sự cống hiến và sự chăm chú”.

Như vậy, Sự tham gia công việc mô tả nhân viên tham gia là người chủ động, năng nổ, kiên trì và tận tâm để hoàn thành các công việc. Bản chất của sự tham gia là sự thưởng thức công việc khi đó những nhân viên nhận thấy công việc của họ hấp dẫn, tràn đầy năng lượng và thách thức.

\subsubsection{Sự kiệt súc}

Theo Leiter và Maslach (2004): Sự kiệt sức đặc trưng bởi kiệt quệ, hoài nghi và không hiệu quả, xuất hiện khi người lao động bị căng thẳng trong công việc trong một thời gian dài. Sự kiệt sức gồm 3 thành phần trạng thái: Sự cạn kiệt (exhaustion) đề cập đến một trạng thái cực kỳ mệt mỏi về thể chất hoặc tinh thần. Thứ hai, Sự hoài nghi (Cynicism) đề cập đến một phản ứng tiêu cực hoặc phản ứng thái quá đối với công việc, thường bao gồm trạng thái mất lý tưởng. Cuối cùng, Sự không hiệu quả (Inefficacy) đề cập đến cảm giác suy giảm về năng lực và năng suất trong công việc.

Kristensen, Hannerz, Høgh, và Borg (2005) trong nghiên cứu của mình lại phân chia khái niệm Sự kiệt sức thành 3 phần khác nhau, bao gồm: Sự kiệt sức cá nhân (Personal burnout); Sự kiệt sức liên quan công việc (Work-related burnout); và Sự kiệt sức liên quan đến khách hàng (Client-related burnout).

Tóm lại Sự kiệt sức là một tình trạng cạn kiệt nguồn lực của nhân viên do tiếp xúc lâu dài với những căng thẳng trong nghề nghiệp vượt quá khả năng tiếp nhận và đối phó của họ. 


\subsection{Các lý thuyết nền tảng}

\subsubsection{Lý thuyết Ý thức bản thân (Self-concept theory)}

Theo tác giả Super (1950), Ý thức bản thân trong nghề nghiệp là sự hợp thành của các thuộc tính tự thân được xem xét bởi cá nhân được coi là có liên quan đến nghề nghiệp. Còn theo tác giả Harter (1999), Ý thức bản thân là một tập hợp các thái độ và nhận thức của một cá nhân về bản thân mình, bao gồm ba yếu tố chính: hình ảnh bản thân, lòng tự trọng và hành vi của chủ thể.

Lý thuyết Ý thức bản thân cung cấp một bối cảnh để hiểu mối quan hệ giữa Ý định nghỉ việc và các yếu tố như cảm nhận về Trách nhiệm xã hội hay cảm nhận về Lãnh đạo đạo đức của nhân viên. Lý thuyết Ý thức bản thân cho thấy rằng nhân viên đòi hỏi bối cảnh xã hội, nhóm hoặc tổ chức lớn hơn để có thể hiểu và thể hiện đầy đủ bản thân từ đó giải tỏa sự mệt mỏi tâm lý của họ (Duchon \& Plowman, 2005). Nói cách khác, Ý thức bản thân của nhân viên được hình thành bởi cảm nhận rằng họ là một phần của nhóm hoặc tổ chức làm việc có trách nhiệm xã hội (Ashforth \& Mael, 1989), do đó làm giảm nguy cơ kiệt sức hoặc căng thẳng về tinh thần. Khi nhân viên nhận thấy tổ chức mà họ làm việc có hoạt động thực thi trách nhiệm xã hội tốt thì cảm giác thuộc về tổ chức có uy tín có thể nâng cao ý thức bản thân của họ và thái độ làm việc của họ trở nên tích cực hơn. Bên cạnh đó, ý thức bản thân còn bị tác động và ảnh hưởng bởi yếu tố cảm nhận về lãnh đạo đạo đức dẫn đến người nhân viên cảm nhận được ý nghĩa công việc mà họ đang làm từ đó người nhân viên sẽ thể hiện sự gia tăng của động lực, nỗ lực và hành vi sản xuất của họ (Piccolo, Greenbaum, Den Hartog, \& Folger, 2010).

\subsubsection{Lý thuyết bảo tồn nguồn lục của Hobfoll (1989)}

Lý thuyết Bảo tồn nguồn lực (Conservation of Resources - COR) do tác giả Hobfoll đề xuất vào năm 1989. COR bao gồm hai nguyên tắc: Nguyên tắc đầu tiên được gọi là tính ưu việt của tổn thất nguồn lực. Nguyên tắc này cho rằng con người sẽ có cảm giác thiệt hại hơn khi mất nguồn lực so với khi có được nguồn lực. Nguyên tắc thứ hai được gọi là đầu tư nguồn lực. Nguyên tắc này cho rằng mọi người sẽ có xu hướng đầu tư nguồn lực để đối phó với tổn thất nguồn lực, phục hồi từ tổn thất và để có được nguồn lực.

Lý thuyết COR là một lý thuyết rất tốt để giải thích vai trò trung gian của Sự kiệt sức và Sự tham gia công việc trong mối quan hệ giữa Trách nhiệm xã hội và Lãnh đạo đạo đức với Ý định nghỉ việc. Từ quan điểm đạo đức, trong bối cảnh nhân viên có nhiều nguồn lực hơn mà không phải giải quyết các yêu cầu về đạo đức, do công ty có nền tảng trách nhiệm xã hội tốt cũng như chất lượng lãnh đạo cao, thì tâm trạng của nhân viên khi làm việc có thể được cải thiện (e.g., Cohen \& Wills, 1985; Schaufeli \& Bakker, 2004) và họ sẽ sẵn sàng tham gia công việc hơn, gắn bó hơn với công ty. Ngược lại, khi nhân viên mất nguồn lực về mặt tâm lý do phải đối phó với những lo ngại về những vấn đề đạo đức tại nơi làm việc, họ có nhiều khả năng cảm thấy dễ bị tổn thương và tiêu cực với mọi thứ tại nơi làm việc (ví dụ như kiệt sức hoặc từ bỏ công việc) (Rappaport, 1981; Siddiqi, 2013), do đó thúc đẩy ý định rời khỏi tổ chức của họ.

\subsection{Các nghiên cứu liên quan và đề xuất mô hình nghiên cứu}

\subsubsection{Các nghiên cứu có liên quan}

Cho đến nay có khá nhiều nghiên cứu về mối quan hệ giữa Trách nhiệm xã hội và Ý định nghỉ việc cả trực tiếp lẫn gián tiếp, như các nghiên cứu của Chaudhary (2017), Liu và Lin (2017), Sarfraz và cộng sự (2018), các tác giả đã chứng minh được mối quan hệ trực tiếp giữa Trách nhiệm xã hội và Ý định nghỉ việc hoặc gián tiếp thông qua các biến trung gian hòa giải cho mối quan hệ giữa Trách nhiệm xã hội và Ý định nghỉ việc.

Về mối quan hệ giữa Lãnh đạo và Ý định nghỉ việc cũng có khá nhiều nghiên cứu như 
nghiên cứu của Laschinger và Fida (2014) nghiên cứu về mối quan hệ giữa Lãnh đạo xác thực (một thành phần quan trọng của lãnh đạo đạo đức) và Ý định nghỉ việc thông qua Sự ngược đãi liên quan công việc và Sự kiệt sức. Nghiên cứu của Azanza, Moriano, Molero, và Mangin (2015) thông qua trung gian là Sự tham gia công việc và Nhận dạng nhóm làm việc để xem xét mối quan hệ giữa lãnh đạo xác thực và ý định nghỉ.

Hầu hết các nghiên cứu về mối quan hệ giữa Sự tham gia công việc và Sự kiệt sức với Ý định nghỉ việc đều sử dụng mô hình nhu cầu nguồn lực công việc JD-R (job resources-demands) phát triển từ lý thuyết COR. Mô hình JD-R là nền tảng để xây dựng mối tương quan giữa Sự tham gia công việc và Sự kiệt sức với Kết quả đầu ra của nhân viên (work outcome), có thể kể đến nghiên cứu của Schaufeli và Bakker (2004) đã sử dụng mô hình để chứng minh mối quan hệ giữa Nhu cầu công việc và Nguồn lực công việc ảnh hưởng đến Ý định nghỉ việc thông qua Sự tham gia công việc và Sự kiệt sức; còn tác giả Shemueli, Dolan, Ceretti, và Del Prado (2015) chứng minh mối quan hệ giữa Sự quá tải công việc và Hỗ trợ xã hội với Ý định nghỉ việc thông qua Sự tham gia công việc và Sự kiệt sức.

Nghiên cứu của Lin và Liu (2017) là một trong những nghiên cứu đầu tiên trình bày về lý thuyết Ý thức bản thân hỗ trợ cho sự ảnh hưởng đồng thời của Cảm nhận về Trách nhiệm xã hội và Cảm nhận về Lãnh đạo đạo đức đến Ý định nghỉ việc của nhân viên. Ngoài ra bằng nghiên cứu này tác giả đã đóng góp cho hoạt động nghiên cứu về Đạo đức kinh doanh thong qua việc sử dụng lý thuyết $\mathrm{COR}$ như một khung bao quát để giải thích các vai trò hòa giải Sự tham gia công việc và Sự kiệt sức.

\section{Bảng 1}

Tổng hợp các nghiên cứu về Ý định nghỉ việc

\begin{tabular}{|l|c|c|c|c|c|}
\hline Khái niệm & $\begin{array}{c}\text { Cảm nhận } \\
\text { Trách nhiệm } \\
\text { xã hội }\end{array}$ & $\begin{array}{c}\text { Lãnh } \\
\text { đạo }\end{array}$ & $\begin{array}{c}\text { Sự tham } \\
\text { gia công } \\
\text { việc }\end{array}$ & $\begin{array}{c}\text { Sụ̂ kiệt } \\
\text { sức }\end{array}$ & $\begin{array}{c}\text { Ý định } \\
\text { nghỉ } \\
\text { việc }\end{array}$ \\
\hline Richa Chaudhary (2017) & $\mathrm{X}$ & & $\mathrm{X}$ & & $\mathrm{X}$ \\
\hline $\begin{array}{l}\text { Heather K. Spence Laschinger and } \\
\text { Roberta Fida 2014 }\end{array}$ & & $\mathrm{X}$ & & $\mathrm{X}$ & $\mathrm{X}$ \\
\hline $\begin{array}{l}\text { Garazi Azanza, Juan A. Moriano, } \\
\text { Fernando Molero và Jean-Pierre } \\
\text { Lévy Mangin 2015 }\end{array}$ & & & $\mathrm{X}$ & $\mathrm{X}$ & $\mathrm{X}$ \\
\hline $\begin{array}{l}\text { Wilmar B. Schaufeli và Arnold B. } \\
\text { Bakker 2004 }\end{array}$ & & & $\mathrm{X}$ & $\mathrm{X}$ & $\mathrm{X}$ \\
\hline $\begin{array}{l}\text { Rachel Gabel Shemueli, Simon L. } \\
\text { Dolan, Adriana Suárez Ceretti và } \\
\text { Pamela Nuñez del Prado 2015 }\end{array}$ & & $\mathrm{X}$ & $\mathrm{X}$ & $\mathrm{X}$ & $\mathrm{X}$ \\
\hline $\begin{array}{l}\text { Chieh-Peng Lin và Min-Ling Liu } \\
2017\end{array}$ & $\mathrm{X}$ & & & & \\
\hline
\end{tabular}

Nguồn: Tác giả tổng hợp 


\subsection{2. Đề xuất mô hình và giả thuyết nghiên cứu}

Sau khi thiết lập cơ sở lý thuyết và tổng hợp các nghiên cứu trước đây, tác giả đề xuất mô hình nghiên cứu dựa trên nghiên cứu gốc của tác giả Liu và Lin (2017) như sau:

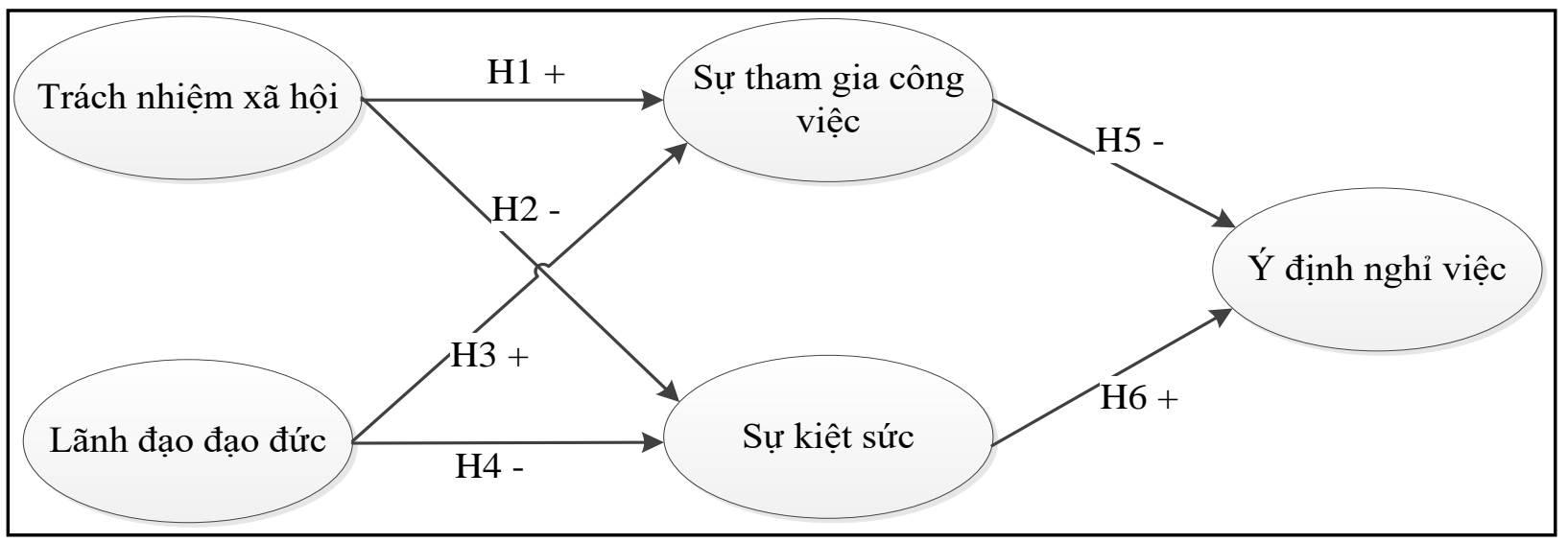

Nguồn: Tác giả đề xuất

Hình 1. Mô hình nghiên cứu đề xuất

Dựa trên các nghiên cứu trước đây về các mối quan hệ giữa các khái niệm Cảm nhận về Trách nhiệm xã hội, Cảm nhận về Lãnh đạo đạo đức, Sự tham gia công việc, Sự kiệt sức và Ý định nghỉ việc làm cơ sở, tác giả đề xuất các giả thuyết nghiên cứu như sau:

Cảm nhận về Trách nhiệm xã hội của nhân viên rất quan trọng bởi vì những cảm nhận này là nền tảng của quan điểm, thái độ và hành vi của nhân viên. Theo đó, những cảm nhận này có thể thúc đẩy nhân viên chủ động hơn trong quyết đinh tham gia vào công việc hoặc làm nhân viên cảm thấy kiệt sức quá trình làm việc. Một doanh nghiệp thực hiện trách nhiệm xã hội tốt hơn có thể giữ được nhiều nhân viên hơn bằng cách tăng cường sự tham gia vào công việc của họ (Gao, Zhang, \& Huo, 2017; Park, Lee, \& Kim, 2018; Rupp et al., 2018). Khi nhân viên nhận thấy rằng công ty của mình thực thi trách nhiệm xã hội tốt thì người nhân viên này sẽ có nhiều động lực hơn để tham gia vào công việc của mình, điều này cho thấy ảnh hưởng tích cực của cảm nhận về Trách nhiệm xã hội của nhân viên đến Sự tham gia công việc. Vì vậy, giả thuyết được đề xuất như sau: nhân viên

H1: Cảm nhận về Trách nhiệm xã hội ảnh hương tích cưc đến Sự tham gia công việc của

Theo lý thuyết Ý thức bản thân, ý thức bản thân của nhân viên được hình thành bởi kiến thức rằng họ là một phần của một nhóm hoặc tổ chức làm việc có trách nhiệm xã hội (Ashforth \& Mael, 1989), do đó làm giảm nguy cơ kiệt sức hoặc căng thẳng về tinh thần. Cụ thể, khi nhân viên cảm nhận rằng được làm việc cho một công ty có hoạt động thực thi trách nhiệm xã hội tốt làm cho công việc của họ đang làm có ý nghĩa hơn sẽ tạo ra tâm lý tích cực cho nhân viên và giảm kiệt sức cảm xúc tiêu cực (tức là kiệt sức) (Raub \& Blunschi, 2014; Yates \& Hollensbe, 2013). Vì vậy, giả thuyết được đề xuất như sau:

\section{H2: Cảm nhận về Trách nhiệm xã hội ảnh hương tiêu cưc đến Sụ kiệt sức của nhân viên}

Theo Piccolo và cộng sự (2010), ý thức bản thân bị tác động và ảnh hưởng bởi yếu tố Lãnh đạo đạo đức. Tương tự như trách nhiệm xã hội, cảm nhận về Lãnh đạo đạo đức giúp cho nhân viên nhận thấy rằng công việc của họ có ý nghĩa hơn từ đó người nhân viên sẽ thể hiện ra sự tăng lên của động lực, nỗ lực và hành vi sản xuất của họ. Các nhà lãnh đạo có tiêu chuẩn cao về đạo đức có thể thúc đẩy sự tham gia công việc của các nhân viên có thái độ tích cực (Ahmad \& Gao, 2018; Azanza et al., 2015; Brown et al., 2005). Vì vậy, giả thuyết được đề xuất như sau: 

nhân viên

H3: Cảm nhận về Lãnh đạo đạo đức ảnh hưởng tích cực đến Sụ tham gia công việc của

Các nghiên cứu trước đây cho rằng lãnh đạo có đạo đức cao sẽ giúp thúc đẩy mối quan hệ gắn bó hơn giữa lãnh đạo và nhân viên dưới quyền (Chughtai, Byrne, \& Flood, 2014; Lambert, Hogan, Barton-Bellessa, \& Jiang, 2012), khi lãnh đạo tạo dựng được niềm tin từ nhân viên và hỗ trợ nhân viên nhiều hơn (Mo \& Shi, 2015; Okpozo, Gong, Ennis, \& Adenuga, 2017) thì các cảm nhận về cảm xúc tiêu cực và sự kiệt sức của nhân viên giảm đi. Vì vậy, giả thuyết được đề xuất như sau:

\section{H4: Cảm nhận về Lãnh đạo đạo đức ảnh hương tiêu cực đến Sụ kiệt sức của nhân viên}

Theo lý thuyết bảo tồn nguồn lực khi nhân viên có đủ nguồn lực, họ sẽ cống hiến cho công việc với khả năng phục hồi nguồn lực tinh thần và tâm lý, từ đó họ sẽ sẵn sàng tham gia công việc hơn, muốn gắn bó hơn và giảm đi mong muốn nghỉ việc (Cohen \& Wills, 1985; Schaufeli \& Bakker, 2004). Ngược lại, khi nhân viên trải qua sự suy giảm về cảm giác tràn đầy năng lượng và nhiệt tình do nguồn lực tâm lý bị lạm dụng hay nói cách khác là họ bị Kiệt sức và trở nên tích cực trong việc tìm kiếm cơ hội việc làm thay thế để khôi phục những cảm xúc đó làm gia tăng mong muốn nghỉ việc (Rappaport, 1981; Siddiqi, 2013). Vì vậy, giả thuyết được đề xuất như sau:

\section{H5: Sụ tham gia công việc ảnh hưởng tiêu cưc đến Ý định nghỉ việc của nhân viên}

\section{H6: Sự kiệt sức ảnh hưởng tích cực đến Ý định nghi việc của nhân viên}

\section{Phương pháp nghiên cứu}

\subsection{Thang đo nghiên cúu}

Dựa trên kết quả tổng kết lý thuyết trước đây về các khái niệm nghiên cứu, tác giả kế thừa từ thang đo của $\mathrm{Ng}$ và cộng sự (2018) và Liu và Lin (2017) và thực hiện thảo luận nhóm với 5 chuyên gia làm quản lý và làm việc lâu năm có quan tâm đến các yếu tố bậc cao như giá trị đạo đức, phát triển bền vững và họ làm việc tại các doanh nghiệp đang có nhiều hoạt động xã hội để tiến hành điều chỉnh và xây dựng thang đo chính thức cho phù hợp với bối cảnh nghiên cứu tại Việt Nam. Thang đo Likert 5 điểm được sử dụng cho các khái niệm trong nghiên cứu.

Thang đo cảm nhận về Trách nhiệm xã hội được tác giả kế thừa từ thang đo của $\mathrm{Ng}$ và cộng sự (2018) với 7 biến quan sát, tác giả đã loại bỏ 1 biến quan sát và hiệu chỉnh ngôn từ 3 biến quan sát để rõ ràng dễ hiểu hơn sau khi thực hiện bước nghiên cứu định tính. Tất cả các thang đo còn lại được tác giả kế thừa từ nghiên cứu của Liu và Lin (2017), cụ thể: thang đo Cảm nhận về Lãnh đạo đạo đức có 6 biến quan sát, tác giả hiệu chỉnh ngôn từ 1 biến quan sát để rõ ràng dể hiểu hơn; thang đo Sự tham gia công việc có 6 biến quan sát, tác giả hiệu chỉnh ngôn từ 1 biến quan sát để rõ ràng dễ hiểu hơn; thang đo Sự kiệt sức có 4 biến quan sát, tác giả hiệu chỉnh ngôn từ 1 biến quan sát để rõ ràng dễ hiểu hơn; và thang đo Ý định nghỉ việc có 4 biến quan sát, tác giả hiệu chỉnh ngôn từ 1 biến quan sát để rõ ràng dễ hiểu hơn.

\subsection{Mẫu nghiên cứu}

Nghiên cứu thực hiện chọn mẫu phi xác suất và lấy mẫu thuận tiện. Về hình thức khảo sát: tác giả sử dụng đồng thời hình thức phát phiếu khảo sát trực tiếp đến đối tượng được khảo sát và khảo sát trên mạng internet thông qua công cụ goole form theo đường link https://forms.gle/trhCgmQNjs7umhD76. Đã có 258 đáp viên tham gia khảo sát thông qua hình thức phát phiếu khảo sát trực tiếp và khảo sát trực tuyến, sau quá trình làm sạch dữ liệu, có 248 mẫu khảo sát đạt yêu cầu được tác giả đưa vào phân tích định lượng. 


\section{Kết quả và thảo luận}

\subsection{Kết quả nghiên cúu}

\subsubsection{Mô tả mẫu nghiên cưu}

Kết quả phân tích thống kê cho thấy: đáp viên chủ yếu là nữ với tỷ lệ $63 \%$, so với nam là 37\%; Độ tuổi của đáp viên chủ yếu từ 25 đến 40 tuổi, chiếm tỷ lệ $90 \%$; Về trình độ học vấn, đáp viên chủ yếu có trình độ từ đại học trở lên chiếm tỷ lệ $95 \%$; Tỷ lệ về vị trí làm việc của các đáp viên khá cân bằng với $43 \%$ là quản lý và $57 \%$ là nhân viên; Các đáp viên chủ yếu có kinh nghiệm làm việc từ 3 năm trở lên với tỷ lệ $96 \%$. Kết quả chi tiết như sau:

\section{Bảng 2}

Thống kê mẫu nghiên cứu

\begin{tabular}{|l|l|r|r|}
\hline \multicolumn{2}{|c|}{ Thông tin mẫu khảo sát } & Tần số & Tỷ lệ (\%) \\
\hline \multirow{3}{*}{ Giới tính } & Nam & 91 & $37 \%$ \\
\cline { 2 - 4 } & Nữ & 157 & $63 \%$ \\
\hline \multirow{4}{*}{ Độ tuổi } & Dưới 25 & 7 & $3 \%$ \\
\cline { 2 - 4 } & Từ 25 đến 40 & 222 & $90 \%$ \\
\cline { 2 - 4 } & Trên 40 & 19 & $7 \%$ \\
\hline \multirow{5}{*}{ Trình độ học vấn } & Dưới Cao đẳng & 0 & $0 \%$ \\
\cline { 2 - 5 } & Cao đẳng & 11 & $5 \%$ \\
\cline { 2 - 4 } & Đại học & 186 & $75 \%$ \\
\cline { 2 - 5 } & Sau Đại học & 51 & $20 \%$ \\
\hline \multirow{2}{*}{ Vị trí công việc } & Nhân viên & 140 & $57 \%$ \\
\cline { 2 - 5 } & Quản lý & 108 & $43 \%$ \\
\hline \multirow{3}{*}{ Kinh nghiệm làm việc } & Dưới 3 năm & 10 & $4 \%$ \\
\cline { 2 - 5 } & Từ 3 đến 10 năm & 137 & $55 \%$ \\
\cline { 2 - 5 } & Trên 10 năm & 101 & $41 \%$ \\
\hline
\end{tabular}

Nguồn: Tác giả phân tích SPSS

\subsubsection{Kiểm định thang đo nghiên cưu}

Kết quả kiểm định độ tin cậy Cronbach's Alpha và EFA cho các tiêu chí Eigenvalues, phương sai trích, KMO \& Bartlett's Test và hệ số tải nhân tố của các thang đo là hoàn toàn phù hợp. Kết quả Ma trận xoay nhân tố từ bảng 3 cho thấy, tất cả các biến quan sát được rút trích thành 5 nhân tố theo cùng nhóm nhân tố. Từ kết quả này cho thấy so với mô hình nghiên cứu mà tác giả đã đề xuất không phát sinh nhân tố mới. 


\section{Bảng 3}

Bảng ma trận xoay nhân tố

\begin{tabular}{|c|c|c|c|c|c|c|c|}
\hline \multirow{2}{*}{\begin{tabular}{l|} 
Khái \\
niệm
\end{tabular}} & \multirow{2}{*}{\multicolumn{2}{|c|}{ Biến quan sát }} & \multicolumn{5}{|c|}{ Nhân tố } \\
\hline & & & 1 & 2 & 3 & 4 & 5 \\
\hline \multirow{6}{*}{$\begin{array}{l}\text { Lãnh } \\
\text { đạo } \\
\text { đạo } \\
\text { đức }\end{array}$} & LD3 & $\begin{array}{l}\text { Lãnh đạo của tôi đưa ra quyết định công bằng và } \\
\text { cân bằng }\end{array}$ & 0.895 & & & & \\
\hline & LD4 & Lãnh đạo của tôi là một người đáng tin cậy & 0.894 & & & & \\
\hline & LD2 & $\begin{array}{l}\text { Trong cuộc } s \text { ống cá nhân, Lãnh đạo của tôi là một } \\
\text { người có đạo đức }\end{array}$ & 0.883 & & & & \\
\hline & LD1 & Lãnh đạo của tôi lắng nghe nhân viên của mình & 0.782 & & & & \\
\hline & LD6 & $\begin{array}{l}\text { Khi đưa ra quyết định, Lãnh đạo của tôi nhấn mạnh } \\
\text { tầm quan trọng của đạo đức }\end{array}$ & 0.731 & & & & \\
\hline & LD5 & $\begin{array}{l}\text { Lãnh đạo của tôi cho rằng thành công không chỉ thể } \\
\text { hiện bời kết quả mà còn là cách thực hiện để đạt } \\
\text { được kết quả }\end{array}$ & 0.651 & & & & \\
\hline \multirow{6}{*}{$\begin{array}{l}\text { Cảm } \\
\text { nhận } \\
\text { về } \\
\text { trách } \\
\text { nhiệm } \\
\text { xã hội }\end{array}$} & TN6 & $\begin{array}{l}\text { Công ty chúng tôi khuyến khích nhân viên của mình } \\
\text { tham gia vào các hoạt động tình nguyện }\end{array}$ & & 0.81 & & & \\
\hline & TN5 & $\begin{array}{l}\text { Công ty chúng tôi đóng góp cho các chiến dịch và } \\
\text { chương trình để phát triển cộng đồng địa phương và } \\
\text { xã hội }\end{array}$ & & 0.772 & & & \\
\hline & $\mathrm{TN} 2$ & $\begin{array}{l}\text { Công ty chúng tôi đầu tư, chăm lo cho sự phát triển } \\
\text { của thế hệ tương lai }\end{array}$ & & 0.748 & & & \\
\hline & $\mathrm{TN} 1$ & $\begin{array}{l}\text { Công ty chúng tôi tham gia vào các hoạt động nhằm } \\
\text { bảo vệ và cải thiện chất lượng môi trường tự nhiên }\end{array}$ & & 0.742 & & & \\
\hline & $\mathrm{TN} 4$ & $\begin{array}{l}\text { Công ty chúng tôi kinh doanh với mục tiêu phát triển } \\
\text { bền vững và quan tâm đến thế hệ tương lai chứ } \\
\text { không chỉ kinh doanh vì lợi nhuận }\end{array}$ & & 0.711 & & & \\
\hline & TN3 & $\begin{array}{l}\text { Công ty chúng tôi thực hiện các chương trình để } \\
\text { giảm thiểu tác động tiêu cực đến môi trường tự } \\
\text { nhiên như kêu gọi nhân viên sử dụng tiết kiệm điện, } \\
\text { nước, giấy in... }\end{array}$ & & 0.684 & & & \\
\hline \multirow{6}{*}{$\begin{array}{l}\text { Sự } \\
\text { tham } \\
\text { gia } \\
\text { công } \\
\text { việc }\end{array}$} & TG4 & Công việc truyền cảm hứng cho tôi & & & 0.781 & & \\
\hline & TG3 & Đối với tôi, công việc của tôi là một thách thức & & & 0.777 & & \\
\hline & TG6 & Tôi thấy mục đích, ý nghĩa của công việc mình làm & & & 0.764 & & \\
\hline & TG2 & Tôi cảm thấy rất sôi nổi, mạnh mẽ trong công việc & & & 0.719 & & \\
\hline & TG5 & Tôi nhiệt tình với công việc của mình & & & 0.699 & & \\
\hline & TG1 & Tôi cảm thấy tràn đầy năng lượng khi làm việc & & & 0.663 & & \\
\hline \multirow{4}{*}{$\begin{array}{l}\text { Sự } \\
\text { kiệt } \\
\text { sức }\end{array}$} & KS3 & Tôi muốn từ bỏ công việc của mình & & & & 0.799 & \\
\hline & KS1 & Tôi cảm thấy chán nản với công việc & & & & 0.799 & \\
\hline & KS4 & $\begin{array}{l}\text { Công việc không giống như tôi hình dung và tôi thấy } \\
\text { rất thất vọng }\end{array}$ & & & & 0.778 & \\
\hline & $\mathrm{KS} 2$ & Tôi thấy như bị nhấn chìm trong công việc của mình & & & & 0.761 & \\
\hline \multirow{3}{*}{$\begin{array}{l}\text { Ý } \\
\text { định } \\
\text { nghỉ } \\
\text { việc }\end{array}$} & NV2 & $\begin{array}{l}\text { Tôi nghiêm túc khi nghĩ về việc chuyển sang công } \\
\text { ty khác }\end{array}$ & & & & & 0.787 \\
\hline & NV3 & Tôi có ý định chuyển sang công ty khác & & & & & 0.739 \\
\hline & NV4 & $\begin{array}{l}\text { Tôi sẽ rời khỏi công ty ngay khi tôi tìm được một } \\
\text { công việc tốt hơn }\end{array}$ & & & & & 0.641 \\
\hline
\end{tabular}

Nguồn: Tác giả phân tích SPSS

Kết quả CFA từ bảng 4 bên dưới cho thấy thang đo đã đáp ứng yêu cầu về độ tin cậy Cronbach alpha $(\mathrm{CA}>0.5)$, độ tin cậy tổng hợp $(\mathrm{CR}>0.8)$ và phương sai trích $(\mathrm{AVE}>0.5)$. 


\section{Bảng 4}

Bảng kiểm định các khái niệm trong mô hình

\begin{tabular}{|l|r|r|r|l|}
\hline \multicolumn{1}{|c|}{ Thang đo } & $\begin{array}{c}\text { Độ tin cậy } \\
\text { Cronbach } \\
\text { alpha }\end{array}$ & $\begin{array}{r}\text { Độ tin } \\
\text { cậy tổng } \\
\text { họp }(\text { CR) }\end{array}$ & $\begin{array}{c}\text { Phương } \\
\text { sai trích } \\
\text { (AVE) }\end{array}$ & Giá trị \\
\hline Cảm nhận về Trách nhiệm xã hội & 0.875 & 0.875 & 0.540 & Đạt yêu cầu \\
\hline Lãnh đạo đạo đức & 0.922 & 0.923 & 0.667 & Đạt yêu cầu \\
\hline Sự tham gia công việc & 0.872 & 0.870 & 0.532 & Đạt yêu cầu \\
\hline Sự kiệt sức & 0.865 & 0.867 & 0.624 & Đạt yêu cầu \\
\hline Ý định nghỉ việc & 0.858 & 0.822 & 0.608 & Đạt yêu cầu \\
\hline Chỉ số của mô hình CFA & \multicolumn{3}{|c|}{ CMIN/df = 2.167; TLI=0.905; } & Đạt yêu cầu \\
\hline
\end{tabular}

Nguồn: Tác giả phân tích $\mathrm{AMOS}$ và xử lý bằng Stats tools package

Bên cạnh đó, theo kết quả kiểm định giá trị hội tụ và giá trị phân biệt của các khái niệm trong mô hình nghiên cứu đều có giá trị $\mathrm{P}$ value $<0.05$, đáp ứng được yêu cầu. Như vậy, kết quả phân tích chứng minh thang đo đã đạt độ tin cậy và các khái niệm trong thang đo là các khái niệm phân biệt và phù hợp với dữ liệu thị trường.

\subsubsection{Kiểm định độ phù hợp của mô hình}

Thông qua phân tích SEM (hình 2) cho thấy mô hình phù hợp với dữ liệu thị trường khi các chỉ số Chisquare/df=2.194, TLI=0.903, CFI=0.915 và $\mathrm{RMSEA}=0.070$.

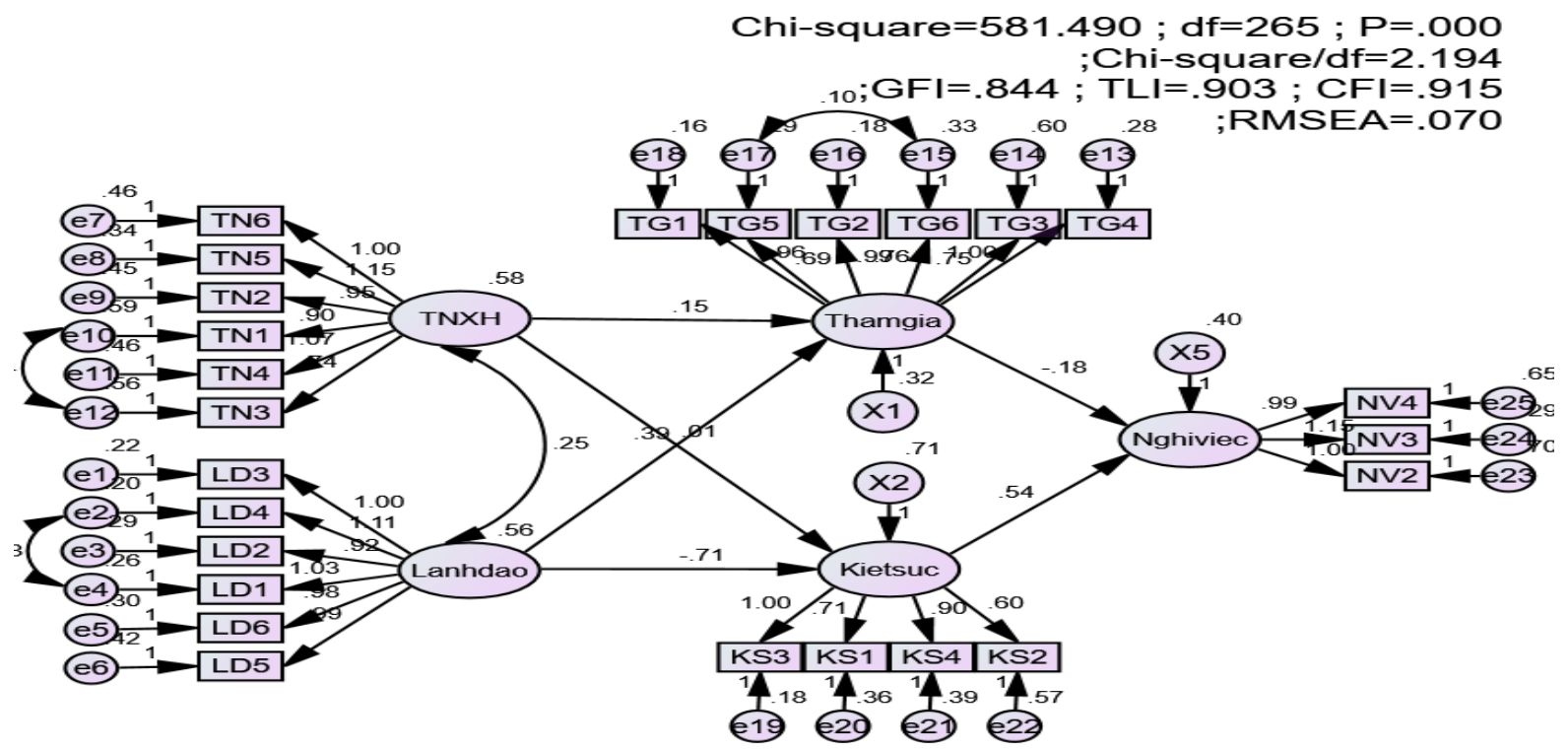

Nguồn: Tác giả phân tích AMOS

Hình 2. Kết quả phân tích SEM

\subsubsection{Kiểm định các giả thuyết nghiên cứu}

Sau khi xác định được mô hình nghiên cứu là hoàn toàn phù hợp với với dữ liệu thị trường và các ước lượng trong mô hình độ tin cậy, tác giả tiến hành kiểm định các giả thuyết nghiên cứu đã được đặt ra ban đầu. 


\section{Bảng 6}

Kết quả kiểm định giả thuyết nghiên cứu

\begin{tabular}{|c|c|c|c|c|c|c|c|c|}
\hline $\begin{array}{c}\text { Giả } \\
\text { thuyết }\end{array}$ & \multicolumn{3}{|c|}{ Mối quan hệ } & $\begin{array}{c}\text { Hệ số } \\
\text { hồi quy }\end{array}$ & S.E. & C.R. & $\begin{array}{c}\text { P- } \\
\text { Values }\end{array}$ & Kết quả \\
\hline $\mathrm{H} 1$ & Thamgia & $<--$ & TNXH & 0.148 & 0.063 & 2.363 & 0.018 & Chấp nhận \\
\hline $\mathrm{H} 2$ & Kietsuc & $<---$ & TNXH & 0.007 & 0.091 & 0.072 & 0.943 & Bác bỏ \\
\hline $\mathrm{H} 3$ & Thamgia & $<---$ & Lanhdao & 0.394 & 0.065 & 6.02 & 0.000 & Chấp nhận \\
\hline $\mathrm{H} 4$ & Kietsuc & $<---$ & Lanhdao & -0.711 & 0.095 & -7.471 & 0.000 & Chấp nhận \\
\hline $\mathrm{H} 5$ & Nghiviec & $<---$ & Thamgia & -0.185 & 0.081 & -2.277 & 0.023 & Chấp nhận \\
\hline H6 & Nghiviec & $<---$ & Kietsuc & 0.536 & 0.065 & 8.196 & 0.000 & Chấp nhận \\
\hline
\end{tabular}

Nguồn: Tác giả phân tích AMOS

\subsection{Thảo luận kết quả nghiên cứu}

Kết quả thực nghiệm cho thấy yếu tố Cảm nhận về Lãnh đạo đạo đức có ảnh hưởng tích cực Sự tham gia công việc của nhân viên (hệ số beta +0.394 với $\mathrm{p}<0.01$ ), phù hợp với các nghiên cứu trước đây (Ahmad \& Gao, 2018; Lin \& Liu, 2017). Nhà lãnh đạo có thể tác động đến cảm nhận và niềm tin của nhân viên từ đó giúp nhân viên nhận thấy được ý nghĩa của công việc mà họ đang thực hiện và chủ động tham gia mạnh mẽ vào công việc.

Về mối quan giữa Cảm nhận về Lãnh đạo đạo đức và Sự kiệt sức của nhân viên, kết quả nghiên cứu chứng minh ảnh hưởng tiêu cực của Cảm nhận về Lãnh đạo đạo đức đến Sự kiệt sức của nhân viên (hệ số beta -0.711 với $\mathrm{p}<0.01$ ), phù hợp với các nghiên cứu trước đây (Laschinger \& Fida, 2014; Lin \& Liu, 2017). Điều này chứng tỏ nhân viên bị tác động mạnh mẽ bởi nhà Lãnh đạo có đạo đức.

Với yếu tố Cảm nhận về Trách nhiệm xã hội có tác động tích cực đến Sự tham gia công việc (hệ số beta +0.148 với $p<0.05$ ), phù hợp với các nghiên cứu trước đây (Gao et al., 2017; Park et al., 2018; Rupp et al., 2018). Nhân viên khi làm việc cho một doanh nghiệp thực hiện Trách nhiệm xã hội tốt sẽ cảm nhận được sự tác động của các yếu tố này đến ý thức bản thân và từ đó họ sẽ chủ động tham gia vào công việc hơn.

Trong nghiên cứu thực nghiệm này, tác giả chưa chứng minh được tác động tiêu cực của Cảm nhận về Trách nhiệm xã hội đến Sự kiệt sức của nhân viên (hệ số beta 0.007 với p>0.05).

Cuối cùng thông qua nghiên cứu này, tác giả khẳng định lại ảnh hưởng tiêu cực của Sự tham gia công việc đến Ý định nghỉ việc của nhân viên (hệ số beta -0.185 với $\mathrm{p}<0.05$ ) và ảnh hưởng tích cực của Sự kiệt sức đến Ý định nghỉ việc của nhân viên (hệ số beta +0.536 với $p<0.05$ ), phù hợp với các nghiên cứu trước đây (Lin \& Liu, 2017; Schaufeli \& Bakker, 2004; Shemueli et al., 2015).

\section{Hàm ý quản trị}

Nghiên cứu đã chỉ ra tác động mạnh mẽ của yếu tố Cảm nhận về Lãnh đạo đạo đức đến Sự tham gia công việc và Sự kiệt sức và thông qua đó tác động đến ý định nghỉ việc của nhân viên. Do đó các nhà quản trị cần nâng cao yếu tố lãnh đạo đạo đức để nhân viên có thể cảm nhận đầy đủ yếu tố này trong tổ chức, từ đó nhân viên sẽ có những quan điểm, thái độ và hành vi phù hợp với các giá trị đạo đức của doanh nghiệp, điều này giúp nhân viên tham gia công việc chủ động hơn và giúp họ giảm bớt nguy cơ kiệt sức và thông qua đó giảm bớt ý định nghỉ việc. Một số hành động cụ thể mà nhà lãnh đạo có thể tham khảo để nâng cao phong cách lãnh đạo đạo đức của mình: 
Nhà lãnh đạo cần lắng nghe và hỗ trợ nhân viên nhiều hơn; Nhà lãnh đạo cần đưa ra những quyết định công bằng và cân bằng; và Nhà lãnh đạo cần nhất quán trong việc thể hiện phong cách lãnh đạo đạo đức trong cuộc sống cá nhân, cũng như trong việc ra quyết định và đánh giá kết quả công việc.

Nghiên cứu cũng cho thấy yếu tố cảm nhận về trách nhiệm xã hội của nhân viên đối với hoạt động thực thi trách nhiệm xã hội của doanh nghiệp chưa thực sự tạo ra tác động mạnh mẽ đến nhân viên. Nguyên nhân có thể do các doanh nghiệp Việt Nam chưa gắn việc thực thi trách nhiệm xã hội với chiến lược kinh doanh và năng lực cốt lõi của mình; Còn đối với nhân viên khi nói đến trách nhiệm xã hội của doanh nghiệp thường chỉ nhìn ở các hoạt động thiện nguyện vì vậy yếu tố trách nhiệm xã hội không đủ mạnh mẽ để tác động đến cảm nhận của nhân viên hoặc đơn giản nhân viên cho rằng những hoạt động mà doanh nghiệp đang thực thi không phải là một dạng của trách nhiệm xã hội ( $\mathrm{Ng}$ et al., 2018). Vì vậy, doanh nghiệp nên tổ chức thực hiện trách nhiệm xã hội một cách có chiến lược để giúp nhân viên cảm nhận được tốt hơn yếu tố này và làm giảm đi ý định nghỉ việc của họ, cụ thể: thứ nhất, lồng ghép trách nhiệm xã hội vào tư duy chiến lược và làm cho nó trở thành một thành phần không thể tách rời trong văn hóa doanh nghiệp và các hoạt động hàng ngày; thứ hai nên truyền đạt quan điểm của mình, trong khi tìm kiếm thông tin phản hồi từ các bên liên quan để cho họ biết và lôi cuốn họ.

\section{Hạn chế nghiên cúu và định hướng cho nghiên cúu tiếp}

Thứ nhất, phạm vi nghiên cứu chỉ tập trung tại khu vực thành phố Hồ Chí Minh và áp dụng phương pháp lấy mẫu thuận tiện (phi xác suất) với số lượng mẫu khá hạn chế (248 mẫu). Do đó tính khái quát của nghiên cứu và tính đại diện mẫu thu thập còn hạn chế.

Thứ hai, đối tượng khảo sát của nghiên cứu là nhân viên văn phòng làm cho nghiên cứu có tính khái quát cao hơn nhưng cũng làm giảm đi khả năng ứng dụng lý thuyết của nghiên cứu. Vì vậy, các nghiên cứu tiếp theo cần đi sâu vào nghiên cứu ở một số lĩnh vực ngành nghề cụ thể.

\section{Tài liệu tham khảo}

Aguilera, R. V., Rupp, D. E., Williams, C. A., \& Ganapathi, J. (2007). Putting the S back in corporate social responsibility: A multileval theory of social change in organizations. Academy of Management Review, 32(3), 836-863.

Ahmad, I., \& Gao, Y. (2018). Ethical leadership and work engagement. Management Decision, 56(9), 1991-2005. doi:10.1108/MD-02-2017-0107

Ashforth, B. E., \& Mael, F. (1989). Social identity theory and the organization. Academy of Management Review, 14(1), 20-39.

Azanza, G., Moriano, J. A., Molero, F., \& Mangin, J. P. L. (2015). The effects of authentic leadership on turnover intention. Leadership \& Organization Development Journal, 36(8), 955-971.

Brown, M. E., \& Treviño, L. K. (2006). Ethical leadership: A review and future directions. The Leadership Quarterly, 17(6), 595-616. doi:10.1016/j.leaqua.2006.10.004

Brown, M. E., Treviño, L. K., \& Harrison, D. A. (2005). Ethical leadership: A social learning perspective for construct development and testing. Organizational Behavior and Human Decision Processes, 97(2), 117-134. doi:10.1016/j.obhdp.2005.03.002 
Carroll, A. (1991). The pyramid of corporate social responsibility: Toward the moral management of organizational stakeholders. Business Horizons, 34, 39-48.

Chaudhary, R. (2017). CSR and turnover intentions: Examining the underlying psychological mechanisms. Social Responsibility Journal, 13(3), 643-660.

Chughtai, A., Byrne, M., \& Flood, B. (2014). Linking ethical leadership to employee well-being: The role of trust in supervisor. Journal of Business Ethics, 128(3), 653-663.

Cohen, S., \& Wills, T. A. (1985). Stress, social support \& the buffering hypothesis. Psychological Bulletin, 98(2), 310-357.

Demirtas, O., \& Akdogan, A. A. (2014). The effect of ethical leadership behavior on ethical climate, turnover intention, and affective commitment. Journal of Business Ethics, 130(1), 59-67. doi:10.1007/s10551-014-2196-6

Duchon, D., \& Plowman, D. A. (2005). Nurturing the spirit at work: Impact on work unit performance. The Leadership Quarterly, 16(5), 807-833.

Gao, Y., Zhang, D., \& Huo, Y. (2017). Corporate social responsibility and work engagement: Testing a moderated mediation model. Journal of Business and Psychology, 33(1), 661-673. doi:10.1007/s10869-017-9517-6

Harter, S. (1999). The construction of the self: A developmental perspective. New York, NY: The Guilford Press.

Hobfoll, S. E. (1989). Conservation of resources: A new attempt at conceptualizing stress. American Psychologist, 44(3), 513-524.

Hollingworth, D., \& Valentine, S. (2014). Corporate social responsibility, continuous process improvement orientation, organizational commitment and turnover intentions. International Journal of Quality \& Reliability Management, 31(6), 629-651.

Khan, M. S., Khan, I., Kundi, G. M., Khan, S., Nawaz, A., Khan, F., \& Yar, N. B. (2014). The impact of job satisfaction and organizational commitment on the intention to leave among the academicians. International Journal of Academic Research in Business and Social Sciences, 4(2), 114-131.

Kristensen, T. S., Hannerz, H., Høgh, A., \& Borg, V. (2005). The copenhagen psychosocial questionnaire - A tool for the assessment and improvement of the psychosocial work environment. Scandinavian Journal of Work, Environment \& Health, 31(6), 438-449. doi:10.5271/sjweh.948

Lambert, E. G., Hogan, N. L., Barton-Bellessa, S. M., \& Jiang, S. (2012). Examining the relationship. Criminal Justice and Behavior, 39(7), 938-957.

Laschinger, H. K. S., \& Fida, R. (2014). A time-lagged analysis of the effect of authentic leadership on workplace bullying, burnout, and occupational turnover intentions. European Journal of Work and Organizational Psychology, 23(5), 739-753.

Leiter, M. P., \& Maslach, C. (2004). Areas of worklife: A structured approach to organizational predictors of job burnout. Emotional and Physiological Processes and Positive Intervention Strategies, 3, 91-134.

Lin, C. P., \& Liu, L. M. (2017). Examining the effects of corporate social responsibility and ethical leadership on turnover intention. Personnel Review, 46(3), 526-550. 
Lu, L., Lu, A. C. C, Gursoy, D., \& Neale, N. R. (2016). Work engagement, job satisfaction, and turnover intentions. International Journal of Contemporary Hospitality Management, 28(4), 737-761.

Martin, A., \& Roodt, G. (2008). Perceptions of organisational commitment, job satisfactionand turnover intentions in a post-merger South African tertiary institution. SA Journal of Industrial Psychology, 34(1), 23-31.

Maslach, C., \& Leiter, M. P. (2008). Early predictors of job burnout and engagement. Journal of Applied Psychology, 93(3), 498-512.

Mo, S., \& Shi, J. (2015). Linking ethical leadership to employee burnout, workplace deviance and performance: Testing the mediating roles of trust in leader and surface acting. Journal of Business Ethics, 144(2), 293-303. doi:10.1007/s10551-015-2821-z

Ng, T. W. H, Yam, K. C., \& Aguinis, H. (2018). Employee perceptions of corporate social responsibility: Effects on pride, embeddedness, and turnover in press personnel psychology. Personnel Psychology, 72(1), 1-31. doi:10.1111/peps.12294

Okpozo, A. Z., Gong, T., Ennis, M. C., \& Adenuga, B. (2017). Investigating the impact of ethical leadership on aspects of burnout. Leadership \& Organization Development Journal, 38(8), 1128-1143. doi:10.1108/LODJ-09-2016-0224

Palanski, M., Avey, J. B., \& Jiraporn, N. (2014). The effects of ethical leadership and abusive supervision on job search behaviors in the turnover process. Journal Business Ethics, 121(1), 135-146. doi:10.1007/s10551-013-1690-6

Park, S. Y., Lee, C. K., \& Kim, H. (2018). The influence of corporate social responsibility on travel responsibility on travel. International Journal of Contemporary Hospitality Management, 30(1), 178-196.

Piccolo, R. F., Greenbaum, R., Den Hartog, D. N., \& Folger, R. (2010). The relationship between ethical leadership and core job characteristics. Journal of Organizational Behavior, 31(2), 259-278.

Rappaport, J. (1981). In praise of paradox: A social policy of empowerment over prevention. American Journal of Community Psychology, 9(1), 1-25.

Raub, S., \& Blunschi, S. (2014). The power of meaningful work: How awareness of CSR initiatives fosters task significance \& positive work outcomes in service employees. Cornell Hospitality Quarterly, 55(1), 10-18.

Rupp, D. E., Shao, R., Skarlicki, D. P., Paddock, E. L., Kim, T.-Y., \& Nadisic, T. (2018). Corporate social responsibility and employee engagement: The moderating role of CSR-specific relative autonomy and individualism. Journal of Organizational Behavior, 39(5), 559-579. doi10.1002/job.2282

Sarfraz, M., Qun, W., Abdullah, M. I., \& Alvi, A. T. (2018). Employees' perception of corporate social responsibility impact on employee outcomes: Mediating role of organizational justice for small and medium enterprises (SMEs). Sustainability, 10(7), 24-29. doi:10.3390/su10072429

Schaufeli, W. B., \& Bakker, A. B. (2004). Job demands, job resources, and their relationship with burnout and engagement: A multi-sample study. Journal of Organizational Behavior, 25(3), 293-315. doi:10.1002/job.248 
Schaufeli, W. B., Salanova, M., Gonzalez-Roma, V., \& Bakker, A. B. (2002). The measurement of engagement and burnout: A two sample confirmatory factor analytic approach. Journal of Happiness Studies, 3(1), 71-92. doi:10.1023/A:1015630930326

Shemueli, R. G., Dolan, S. L., Ceretti, A. S., \& Del Prado, P. N. (2015). Burnout and engagement as mediators in the relationship between work characteristics and turnover intentions across two Ibero-American nations. Stress and Health, 32(5), 597-606. doi:10.1002/smi.2667

Siddiqi, M. A. (2013). Examining work engagement as a precursor to turnover intentions of service employees. Business and Management, 5(4), 118-132.

Super, D. (1950). Vocational adjustment: Implementing a self-concept. The Career Development Quaterly, 36, 88-92.

Turker, D. (2009). How corporate social responsibility influences organizational commitment. Journal of Business Ethics, 89(2), 189-204.

Vitaliano, D. (2010). Corporate social responsibility and labor turnover. The International Journal of Business in Society, 10(5), 563-573.

Yates, M., \& Hollensbe, E. (2013). On the positives of peripheral corporate social responsibility. Industrial and Organizational Psychology, 6(4), 368-372. 